\title{
Research Progress of HOXA13/HOTTIP Gene and Digestive Tract Cancer
}

\author{
Meiling Fang1, Yuantai Wan ${ }^{1 *}$, Jinjun $\mathrm{Li}^{2}$ \\ ${ }^{1}$ Department of Gastroenterology, Tianyou Hospital Affiliated to Wuhan University of Science and Technology, Wuhan, China \\ ${ }^{2}$ Department of Surgery, College of Medicine, Wuhan University of Science and Technology, Wuhan, China \\ Email: ${ }^{\star} 1327015410 @ q q . c o m$
}

How to cite this paper: Fang, M.L., Wan, Y.T. and Li, J.J. (2017) Research Progress of HOXA13/HOTTIP Gene and Digestive Tract Cancer. Journal of Cancer Therapy, 8, 1234-1240. https://doi.org/10.4236/jct.2017.813104

Received: November 30, 2017 Accepted: December 16, 2017 Published: December 19, 2017

Copyright ( $) 2017$ by authors and Scientific Research Publishing Inc. This work is licensed under the Creative Commons Attribution International License (CC BY 4.0).

http://creativecommons.org/licenses/by/4.0/

\begin{abstract}
The occurrence and development of digestive tract tumors are mainly caused by the interaction of genetic and environmental factors, multiple incentives, multiple genes involved, and multi-step regulation. With the development of gene sequencing technology, precise treatment of tumor era has arrived. Studies have shown that the HOXA13 gene in the homeobox gene is abnormally expressed in digestive system tumors. Studies have shown that HOXA13 may have a certain relationship with the occurrence, development and prognosis of the tumor, pending the diagnosis and treatment of digestive tract tumors with a new gene target.
\end{abstract}

\section{Keywords}

HOXA13, HOTTIP, Tumor of Digestive Tract

\section{Introduction}

The incidence of esophageal cancer ranks the fifth among all kinds of malignant tumors in China, and the mortality rate ranks the fourth place in [1] [2] [3]. Gastric cancer is one of the most common malignant tumors in the world, with fifth malignant tumors and third death rates. In urban areas, the incidence of gastric cancer is second only to lung cancer and colorectal cancer. In rural areas, the incidence of gastric cancer is only lower than that of lung cancer [4] [5] [6]. Pancreatic cancer is the fourth most common cause of death in men (second only to lung cancer, prostate cancer and colorectal cancer), and is also the fourth leading cause of cancer death in women (second only to lung cancer, breast cancer and colorectal cancer) [7] [8] [9] [10]. Ma Chen et al. [11] show that the incidence of pancreatic cancer is on the rise in China, and pancreatic cancer is still 
one of the major cancers in the short term. Liver cancer is the most heterogeneous and the highest mortality of refractory malignant tumor. The mortality rate of liver cancer in China is the second, second only to lung cancer [12] [13].

\section{HOX Gene Overview}

Homeobox gene (homeoboxgene) was first discovered in Drosophila is a class of genes play an important role in embryonic development and cell differentiation in the people included in the animal, many evidences show that the abnormal expression of HOX may play an important role in diseases including tumor. The conserved sequence of $180-183$ nucleotides in the gene sequence consists of 60 61 conserved amino acid sequences, which are homologous domains or homeobox. HOX gene belongs to class I homeobox gene, which is highly protective. COX gene can be divided into four groups: A, B, C and D according to the sequence similarity and the position on chromosome. Located in 7 (HOXA), 17 (HOXB), 12 (HOXC), 2 (HOXD) on chromosome, according to the similarity on the chromosome and gene position between ethnic sequences can be divided into 13 groups, the HOX gene of mammalian animal has been identified a total of 39. Homeobox gene encodes a highly conserved HOX protein, which regulates DNA gene expression, cell differentiation and morphogenesis by binding to the third helix of amino acid and homologous protein segment of $\mathrm{N}$ terminus. The expression of HOX gene with time and space is linear, if its abnormal expression will lead to individual development and organ formation of abnormal morphology in the process, and can lead to the formation of tumor cell malignant transformation [14]-[25].

\section{Research Status of HOXA13/H0TTIP}

HOXA13 gene is a member of the HOXA family, 5 is located at the end of chromosome 7, under normal circumstances, plays an important role in the formation and organization of blood vessels on the embryonic limb development and reproductive system. HOTTIP (transcript of a HOXA gene family terminal) is a non RNA cancer gene encoding, but also a variety of tumor, HOTTIP silencing in vivo by reducing $\mathrm{Bcl}-2$ and enhance the expression of $\mathrm{Bax}$ inhibited the cell survival pathway, inhibit HOTTIP expression of cyclin cycling and D1 induced cell cycle arrest in G0/G1 phase. Down regulation of HOTTIP can lead to the expression of HOXA13 gene decreased [25] [26] [27] [28] [29]. At present, studies have shown that the HOXA13 signaling pathway by transforming growth factor beta (TGF-beta) increased invasion of gastric cancer cells and transformed epithelial mesenchymal cells between the (EMT) [30]; HOXA13 by HOXA with targeted trans activation of insulin-like growth factor binding protein 3 (IGFBP-3), then [32] and invasion of gastric cancer cells with high carcinogenicity HOXA13; -17 (CDH17) by regulating cad her in expression of activated Wnt-beta catenin signaling pathway. DKK1, cmyc, cyclinD1 and [31] [32] [33] [34] promote gastric cancer cell proliferation. HOXA13 is also associated 
with gastric cancer, liver cancer, pancreatic cancer, esophageal squamous cell carcinoma, and so far, there are few reports in colorectal cancer.

\section{H0XA13/H0TTIP and Digestive Tract Tumor}

1) Abnormal expression of HOXA13/HOTTIP gene and esophageal squamous cell carcinoma

Yan Wanpu [35] for the expression of HOXA13 gene related to embryo research in esophageal squamous cell carcinoma and its impact on the prognosis, they screened 39 cases without chemotherapy in stage a (T3N0M0) patients on the expression were detected by immunohistochemistry SP protein HOXA13 gene. The results suggest that HOXA13 protein in esophageal squamous cell carcinoma positive expression rate was $61.5 \%$, and the HOXA13 expression and age, gender, tumor location, histological differentiation, no significant correlation $(\mathrm{P}>0.05)$, using the Kaplan-Meier analysis shows that the positive expression of HOXA13 were HOXA13 negative expression of disease-free survival was significantly shorter $(\mathrm{P}<0.05)$. The increased expression of HOXA13 it might be a predictor of stage a esophageal squamous cell carcinoma patients. Shen Lu-Yan [36], Ma Ruo-La [37] and other studies also support this view.

2) Abnormal expression of HOXA13/HOTTIP gene and gastric cancer

Han Yang [38] and screened 132 without preoperative chemotherapy of gastric cancer patients were collected after operation of carcinoma tissue and paracancerous normal mucosa, immunohistochemical staining showed that the expression of HOXA13 in gastric cancer was significantly higher than that of adjacent normal mucosa $(\mathrm{P}<0.001)$, and the expression of HOXA13 in patients with significant overall survival the rate of disease-free survival and the expression of HOXA13 was significantly lower than that of patients (the former HR 3.331.95\% CI $1.722-6.442 \mathrm{p}<0.001$ HR $3.28995 \%$ CI; the latter, $1.703-6.351, \mathrm{p}<0.001$ ), shows that the expression of HOXA13 is likely associated with tumor invasive phenotype. Furthermore, Chang Shuai [26] in 50 cases of gastric cancer tissues and corresponding normal tissues by qRT-PCR technique and statistical analysis (SPSS test, Spear T software data, man rank correlation test), the results showed that HOTTIP and HOXA13 in gastric cancer tissue than in adjacent normal tissues was significantly upregulated in the low; the differentiation of gastric carcinoma, the expression of HOTTIP and HOXA13 were higher $(\mathrm{P}<0.05)$; high TNM stage $(\mathrm{P}<0.05)$ and the lymph metastasis $(\mathrm{P}<0.01)$, more than [39] [40] [41] [42] and the results of related studies suggest that HOTTIP and HOXA13 with the progress of gastric cancer has a high correlation, correlation.

3) Abnormal expression of HOXA13/HOTTIP gene and pancreatic ductal carcinoma

Li Zhihua [29] collected 8 patients with a diagnosis of pancreatic ductal adenocarcinoma tissues and 4 pancreatic tissue, transcription level was measured by quantitative real-time PCR and HOTTIP HOXA13 of the sample; down to assess the role of HOTTIP and HOXA13 in the invasion and proliferation of epithelial 
cells and mesenchymal transition of the target to in vitro. The results showed that the expression of HOTTIP in pancreatic ductal carcinoma than in normal pancreatic tissue; immunohistochemical results suggest that high expression of HOXA13 and pancreatic ductal adenocarcinoma with lymph node metastasis, survival rate and adverse tissue variation decreased, therefore, the HOTTIP/ HOXA13 axis may be a therapeutic target and molecular markers of pancreatic ductal adenocarcinoma.

4) Abnormal expression of HOXA13/HOTTIP gene and hepatocellular carcinoma

Quagliata Luca [28] collected 52 without any treatment of hepatocellular carcinoma with tumor tissues and corresponding non tumor tissues, and using hepatoma derived cell function test results ( $\mathrm{HuH}-6$ and $\mathrm{HuH}-7)$ of high expression obtained in non tumor tissue HOTTIP and HOXA13 in HCC specimens were matched $(\mathrm{P}<0.05)$, they on tumor metastasis rate and overall survival rate of patients that HOXA13 expression as an independent predictor for Kaplan Maier analysis (relative risk (relative risk) 2.345, $\mathrm{P}=0.042$ ). Overall survival in univariate analysis showed that liver cell cancer and HOXA13 multiple liver as a predictor (relative risk 2.740, the former: $\mathrm{P}=0.014$; the latter: relative risk $2.181, \mathrm{P}=$ 0.050 ), the above data may said marker HOXA13 can be used as a molecular biological diagnosis of liver cancer patients with clinical signs and judging results.

5) HOX gene and colon cancer

A lot of research on the relationship between HOX gene and colorectal cancer at present, studies have shown that the development of colon cancer and HOXB7, HPXB8, HOXB9 and HOXA5 expression of homeobox genes are associated with HOXA13, but the research is very little, need to be further studied.

\section{Prospect}

In other tumor systems of the human body, the research of HOXA13 gene are many, such as prostate cancer, ovarian cancer [43] [44], in T cell acute lymphoblastic leukemia [45]. The pathogenesis of HOX gene family and tumor has received increasing attention, especially in HOXA13/HOTTIP, but the tumor pathogenesis and signaling pathway is still unclear further research is needed, HOXA13/HOTTIP may be for early diagnosis of neoplastic diseases and provide molecular biological markers in predicting the prognosis markers. At the same time, HOXA13 is an independent factor that promotes tumor proliferation, invasion and metastasis, suggesting that it may serve as an effective target for tumor gene targeted therapy.

\section{Summary}

The current research on the digestive tract and other tumors HOXA13 lacks of mechanism of the occurrence, development and prognosis of tumor, and clarifying the HOXA13 still needs further research and discussion, with the deepening of the study, HOXA13 needs to become tumor diagnosis and treatment to 
provide new targets. Of course, environmental factors play an important role in the development of tumors. Modern medical research shows that the occurrence of cancer is the result of the interaction of environmental factors and genetic factors. The chemical, biological and physical factors in the environment and their interaction with each other intertwined, can produce intermittent or sustained repeated attacks on the human body.

\section{References}

[1] Yang, X.P., Liu, W.W., et al. (2015) Epidemiological Investigation of 1108 Patients with Esophageal Cancer. Chinese Journal of Gastrointestinal Endoscopy, 2, 118-121.

[2] He, J. and Shao, K. (2011) Epidemiological Status, Diagnosis and Treatment of Esophageal Cancer in China and Its Future Strategy. Chinese Journal of Cancer, 21, 501-503.

[3] Fitzgerald, R.C., Di Pietro, M., Ragunath, K., et al. (2014) British Society of Gastroentery Guidelines on the Diagnosis and Management of Barrett's Oesophagus. Gut, 63, 7-42. https://doi.org/10.1136/gutjnl-2013-305372

[4] Zuo, T.T., Zheng, R.S., Zeng, H.M., et al. (2017) Epidemiological Status of Gastric Cancer in China. Chinese Cancer Clinic, 44, 52-58.

[5] Lu, Y.F., Liu, Z.C., Li, Z.H., et al. (2014) Esophageal/Gastric Cancer Screening in High-Risk Populations in Henan Province, China. Asian Pacific Journal of Cancer Prevention, 15, 1419-1422. https://doi.org/10.7314/APJCP.2014.15.3.1419

[6] Yamamoto, K., Tsujinaka, T., Takahashi, T., et al. (2015) Impact of the Japanese Gastric Cancer Screening System on Treatment Outcomes in Gastric Gastrointestinal Stromal Tumor (GIST): An Analysis Based on the GIST Registry. Annals of Surgical Oncology, 22, 232-239. https://doi.org/10.1245/s10434-014-3971-4

[7] Xiang, S., Liang, D.K., et al. (2016) Progress in the Research and Treatment of Pancreatic Cancer in China. Chinese Journal of Cancer, 26, 281-289.

[8] Ma, S.J., Qu, Z.L., Hole, D., et al. (2015) Progress of Pancreatic Cancer Diagnosis and Epidemiological Research. Chinese Combining Traditional Chinese and Western Medicine. Journal of Surgery, 21, 87-92.

[9] Siegel, R., Naishadham, D. and Jemal, A. (2013) Cancer Statistics, 2013. CA: $A$ Cancer Journal for Clinicians, 63, 11-30. https://doi.org/10.3322/caac.21166

[10] StatBite. (2010) U.S. Pancreatic Cancer Rates. Journal of the National Cancer Institute, 102, 18-22.

[11] Ma, C. and Jiang, Y.X. (2013) Trend and Prediction on the Incidence of Pancreatic Cancer in China. Chinese Journal of Epidemiology, 34, 160-163.

[12] Lv, G.S., Chen, L., Wang, H.Y., et al. (2015) The Current Situation and Prospect of Liver Cancer Research in China. Life Sciences, 27, 237-248.

[13] Wang, F.S., Fan, J.G., Zhang, Z., Gao, B. and Wang, H.Y. (2014) The Global Burden of Liver Disease: The Major Impact of China. Hepatology, 60, 2099-2108. https://doi.org/10.1002/hep.27406

[14] Nie, L.H., Mei, C., Wen, J., et al. (2004) Regulation of Homeobox Genes on Vascular Smooth Muscle Cells. Cell Biology Journal, 26, 352-356.

[15] Song, Z. (2003) Homeobox Gene and Tumor. Foreign Medical Oncology Section, 30, 163-166.

[16] Xia, H. (2007) Hox Gene and Tumor Progression Research. International Journal of Stomatology, 34, 412-414. 
[17] Yan, J., Dang, Y., Liu, S.Y., et al. (2016) LncRNA HOTAIR Promotes Cisplatin Resistance in Gastric Cancer by Targeting miR-126 to Activate the PI3K/AKT/MRP1 Genes.

[18] Kyung, J.M., Jong-Jae, P. and Chun, H.J. (2016) Impact of Homeobox Genes in Gastrointestinal Cancer. World Journal of Gastroenterology, 22, 8247-8256.

https://doi.org/10.3748/wjg.v22.i37.8247

[19] Goodman, F.R. and Scambler, P.J. (2001) Human HOX Gene Mutations. Clinical Genetics, 59, 1-11. https://doi.org/10.1034/j.1399-0004.2001.590101.x

[20] Grier, D.G., Thompson, A., Kwasniewska, A., et al. (2005) The Pathophysiology of HOX Genes and Their Role in Cancer. The Journal of Pathology, 205, 154-171. https://doi.org/10.1002/path.1710

[21] Naora Honami, S.S. (2005) Homeobox Gene Expression in Cancer: Insights from Developmental Regulation and Deregulation. European Journal of Cancer, 41, 2428-2437. https://doi.org/10.1016/j.ejca.2005.08.014

[22] Bhatlekar, S. and Fields, J.Z. (2014) Bomanbruce MHOX Genes and Their Role in the Development of Human Cancers. Journal of Molecular Medicine, 92, 811-823. https://doi.org/10.1007/s00109-014-1181-y

[23] Sukumar Saraswati, N.S. (2010) The Hox Genes and Their Roles in Oncogenesis. Nature Reviews Cancer, 10, 361-371. https://doi.org/10.1038/nrc2826

[24] Who, C.S. (2011) Russell Steven Genomic Approaches to Understanding Hox Gene Function. Advances in Genetics, 76, 55-91. https://doi.org/10.1016/B978-0-12-386481-9.00003-1

[25] Wang, S.S., Wuputra, K., Liu, C.J., et al. (2016) Oncogenic Function of the Homeobox A13-Long Noncoding RNA HOTTIP-Insulin Growth Factor-Binding Protein 3 Axis in Human Gastric Cancer. Oncotarget, 7, 36049-36064. https://doi.org/10.18632/oncotarget.9102

[26] Chang, S., Liu, J.S., Guo, S.C., et al. (2016) HOTTIP and HOXA13 Are Oncogenes Associated with Gastric Cancer Progression. Oncology Reports, 35, 3577-3585. https://doi.org/10.3892/or.2016.4743

[27] Lian, Y.F., Cai, Z.L., Gong, H.B., et al. (2016) HOTTIP: A Critical Oncogenic Long Non-Coding RNA in Human Cancers.

[28] Quagliata, L., Matter, M.S., Piscuoglio, S.A., et al. (2014) Long Noncoding RNA HOTTIP/HOXA13 Expression Is Associated with Disease Progression and Predicts Outcome in Hepatocellular Carcinoma Patients. Hepatology, 59, 911-923. https://doi.org/10.1002/hep.26740

[29] Li, Z.H., Zhao, X.H., Zhou, Y., et al. (2015) The Long Non-Coding RNA HOTTIP Promotes Progression and Gemcitabine Resistance by Regulating HOXA13 in Pancreatic Cancer. Journal of Translational Medicine, 13, 442-444. https://doi.org/10.1186/s12967-015-0442-Z

[30] Duan, R., Han, L., Wang, Q.X., et al. (2015) HOXA13 Is a Potential GBM Diagnostic Marker and Promotes Glioma Invasion by Activating the Wnt and TGF-Beta Pathways. Oncotarget, 6, 27778-27793. https://doi.org/10.18632/oncotarget.4813

[31] Qu, L.P., Zhong, Y.M., Zheng, Z., et al. (2017) CDH17 Is a Downstream Effector of HOXA13 in Modulating the Wnt/ $\beta$-Catenin Signaling Pathway in Gastric Cancer. European Review for Medical and Pharmacological Sciences, 21, 1234-1241.

[32] Bartolome, R.A., Barderas, R., Torres, S., et al. (2014) Cadherin-17 Interacts with Alpha 2 Beta 1 Integrin to Regulate Cell Proliferation and Adhesion in Colorectal Cancer Cells Causing Liver Metastasis. Oncogene, 33, 1658-1669.

https://doi.org/10.1038/onc.2013.117 
[33] Qiu, H.B., Zhang, L.Y., Ren, C., Zeng, Z.L., Wu, W.J., Luo, H.Y., Zhou, Z.W. and Xu, R.H. (2013) Targeting CDH17 Suppresses Tumor Progression in Gastric Cancer by Downregulating Wnt/Beta-Catenin Signaling. PLOS ONE, 8, e56959. https://doi.org/10.1371/journal.pone.0056959

[34] Li, R., Yang, H.Q., Xi, H.L., et al. (2017) Inhibition of CDH17 Gene Expression via RNA Interference Reduces Proliferation and Apoptosis of Human MKN28 Gastric Cancer Cells. International Journal of Oncology, 50, 15-22. https://doi.org/10.3892/ijo.2016.3783

[35] Yan, W., Shen, L., Gu, Z., et al. (2009) Expression of Embryonic Gene HOXA13 in Esophageal Squamous Cell Carcinoma and Its Effect on the Prognosis of. The Chinese Journal of Gastrointestinal Surgery, 12, 20-23.

[36] Shen, L.Y. and Chen, K.N. (2011) Exploration of Target Genes of HOXA13 in Esophageal Squamous Cell Carcinoma Cell Line. Cancer Letters, 312, 18-23. https://doi.org/10.1016/j.canlet.2011.07.020

[37] Ma, R.L., Shen, L.Y. and Chen, K.-N. (2014) Coexpression of ANXA2, SOD2 and HOXA13 Predicts Poor Prognosis of Esophageal Squamous Cell Carcinoma. Oncology Reports, 31, 2157-2164. https://doi.org/10.3892/or.2014.3088

[38] Han, Y., Tu, W.W., Wen, Y.G., et al. (2013) Identification and Validation That Up-Expression of HOXA13 Is a Novel Independent Prognostic Marker of a Worse Outcome in Gastric Cancer Based on Immunohistochemistry. Medical Oncology, 30, 564-566. https://doi.org/10.1007/s12032-013-0564-1

[39] Du, M.L., Wang, W.Z., Jin, H., et al. (2015) The Association Analysis of lncRNA HOTAIR Genetic Variants and Gastric Cancer Risk in a Chinese Population. Oncotarget, 6, 31255-31262. https://doi.org/10.18632/oncotarget.5158

[40] Yuan, C., Wang, S.W., Chen, W.I., et al. (2009) A Tumorigenic Homeobox(HOX)gene Expressing Human Gastric Cell Line Derived from Putative Gastric Stem Cell. European Journal of Gastroenterology \& Hepatology, 21, 1016-1023. https://doi.org/10.1097/MEG.0b013e32831d7b9c

[41] Pan, W., Liu, L., Wei, J., et al. (2016) A Functional lncRNA HOTAIR Genetic Variant Contributes to Gastric Cancer Susceptibility. Molecular Carcinogenesis, 55, 90-96. https://doi.org/10.1002/mc.22261

[42] Bayram, S., Yakup, Ü., Taner, S.A., et al. (2015) A Functional HOTAIR rs920778 Polymorphism Does Not Contributes to Gastric Cancer in a Turkish Population: A Case-Control Study. Familial Cancer, 14, 561-567.

https://doi.org/10.1007/s10689-015-9813-0

[43] Zhang, S.R., Yang, J.K., Xie, J.K., et al. (2016) Long Noncoding RNA HOTTIP Contributes to the Progression of Prostate Cancer by Regulating HOXA13. Cellular and Molecular Biology, 62, 84-88.

[44] Kelly, Z., Moller-Levet, C., Sophie, M., Simon, B., Kierzek Andrzej, M., Hardev, P., Morgan, R., Agnieszka, M. and Kavitha, M.T. (2016) The Prognostic Significance of Specific HOX Gene Expression Patterns in Ovarian Cancer. International Journal of Cancer, 139, 1608-1617.

[45] Su, X., Drabkin, H., Clappier, E., Morgado, E., Busson, M., Romana, S., Soulier, J., Berger, R., Bernard, O.A. and Lavau, C. (2006) Transforming Potential of the T-Cell Acute Lymphoblastic Leukemia-Associated Homeobox Genes HOXA13, TLX1, and TLX3. Genes Chromosomes Cancer, 45, 846-855. https://doi.org/10.1002/gcc.20348 